\title{
Compósitos de PET reciclado com fibra de cana-de-açúcar tratada por explosão a vapor
}

\author{
Composites of recycled PET and sugarcane \\ fiber treated by steam explosion
}

\author{
Meriene Gandara ${ }^{1}$, Adilson Roberto Gonçalves ${ }^{2}$, \\ Clodoaldo Saron ${ }^{1}$
}

\footnotetext{
${ }^{1}$ Departamento de Engenharia de Materiais - DEMAR, Escola de Engenharia de Lorena - EEL, Universidade de São Paulo - USP, Polo Urbo Industrial, Gleba AI-6, CEP: 12602-810, Lorena, SP

e-mail: saron@usp.br; merienegandara@gmail.com

${ }^{2}$ Instituto de Pesquisa em Bioenergia - IPBEN - UNESP, Rua 10, 2527, CEP 13500-230 Rio Claro, SP. e-mail:adilson@ipben.unesp.br
}

\section{RESUMO}

A utilização de polímeros termoplásticos reciclados a partir de embalagens para a preparação de compósitos com fibras vegetais é uma alternativa tecnológica de grande potencial que pode conciliar viabilidade econômica com benefícios ambientais. No Brasil, muitos estudos têm sido feitos para a utilização de fibras vegetais em compósitos com matriz polimérica devido à grande disponibilidade de resíduos desses materiais. O País também merece destaque pelo alto índice de reciclagem mecânica de polímeros termoplásticos, principalmente do polímero poli(tereftalato de etileno) (PET). O presente trabalho teve como objetivo a preparação de compósitos por processamento termomecânico a partir de fibra de cana-de-açúcar tratada por explosão a vapor com matriz polimérica de PET e a avaliação do comportamento das propriedades do material em comparação aos compósitos com fibras não tratadas. Apesar das mudanças significativas na morfologia das fibras de cana-de-açúcar com o tratamento por explosão a vapor, não houve diferenças significativas nas propriedades dos compósitos PET/fibra tratada com relação aos compósitos PET/fibra in natura devido à não penetração da matriz polimérica no interior da estrutura da fibra modificada com o tratamento. $\mathrm{O}$ uso de outros tipos de matrizes poliméricas recicladas e adaptações nas operações de processamento dos materiais são alternativas que podem ainda potencializar o uso de fibra de cana-de-açúcar tratada por explosão a vapor na preparação de compósitos poliméricos e contribuir para a produção de materiais ambientalmente sustentáveis.

Palavras-chave: Compósitos poliméricos, fibra de cana-de-açúcar, PET, explosão a vapor.

\section{ABSTRACT}

The use of recycled thermoplastic polymers from packaging and natural fibers for preparation of polymeric composites is a technological alternative of high potential to conciliate economic viability with environmental benefits. In Brazil, several studies have been performed aiming the use of natural fibers in polymeric composites due to the high availability of the agricultural waste. The high index of mechanical recycling of thermoplastic polymers, mainly of the polymer polyethylene terephthalate (PET), also worth mentioning in the Country. This study aimed the preparation of PET/sugarcane fiber composites by thermomechanical processing after treating of the fibers by steam explosion and the evaluation of the composites properties when compared with composites using fibers without treatment. Although of the significant changes in the fiber morphology with steam explosion treatment, there were not significant difference between properties of the composites with treated fibers and the composites with not treated fibers due to the insufficient penetration of the polymeric matrix into structure of the modified fiber after treatment. The use of others recycled polymeric matrix as well as adjustments in the operations for materials processing are alternatives that can improve the use of sugarcane fiber treated by steam explosion in the preparation of polymeric composites, contributing for production of environmentally sustainable materials.

Keywords: Polymeric composites, sugarcane fiber, PET, steam explosion. 


\section{INTRODUÇÃO}

O mercado de embalagens movimenta valores expressivos na economia mundial, sendo que no Brasil em 2014 o valor bruto da produção de embalagens atingiu a cifra de $\mathrm{R} \$ 55,1$ bilhões [1]. Aproximadamente $39 \%$ dos materiais utilizados para embalagens são polímeros, sendo que determinados termoplásticos, como é o caso do poli(tereftalato de etileno) (PET), merecem destaque tanto pelo volume de produção quanto pelos problemas gerados com o seu descarte irregular [1,2]. As embalagens de bebidas apresentam vida útil muito curta, considerando desde a sua produção, envaze, até o descarte como resíduo sólido. Entretanto, a mesma embalagem no meio ambiente pode demorar anos até ser completamente decomposta [3].

A utilização de resíduos de polímeros termoplásticos provenientes de embalagens para novas aplicações por meio da reciclagem mecânica é uma alternativa interessante que pode conciliar aspectos econômicos com benefícios ambientais [4]. Neste sentido, o estudo para a adequação e modificação dos materiais poliméricos é um ponto de grande importância. A aplicação de materiais lignocelulósicos como um componente de reforço em compósitos poliméricos, principalmente na indústria automobilísticas, tem chamado a atenção por causa de seus baixos preço e peso específico [5-7]. Reforços lignocelulósicos oferecem várias vantagens em relação aos inorgânicos, como baixa densidade, alta deformabilidade, baixa abrasividade aos moldes e aos equipamentos de mistura, apresentando ainda baixo custo [8,9]. Deste modo, a reciclagem de resíduos termoplásticos de embalagens na forma de compósitos com fibras vegetais apresenta grande potencial de viabilidade técnica e econômica.

Dentre as fibras naturais, o bagaço de cana-de-açúcar merece destaque pelo seu volume gerado em função do histórico do Brasil no setor sucroalcooleiro. Responsável por mais da metade do açúcar comercializado no mundo, o País deve alcançar taxa média de aumento da produção de $3,25 \%$, até $2018 / 19$, e colher 47,34 milhões de toneladas do produto, o que corresponde a um acréscimo de 14,6 milhões de toneladas em relação ao período 2007/2008. Para as exportações, o volume previsto para 2019 é de 32,6 milhões de toneladas $[10,11]$.

A biomassa vegetal é constituída por celulose, polioses, lignina, pequenas quantidades de extrativos e sais minerais, apresentando uma estrutura lamelar na qual se distribuem seus componentes. A celulose e a hemicelulose predominam na região da parede celular e a lignina distribui-se por toda estrutura, apresentando máxima concentração na lamela média [12]. Os processos de transformação das frações lignocelulósicas são limitados pela estrutura da biomassa. Geralmente, é necessária a ruptura do complexo lignina-celulosehemicelulose ou a remoção de cada fração por técnicas de pré-tratamento e deslignificação [13]. Métodos de modificação das propriedades físicas ou químicas das fibras vegetais têm sido estudados e desenvolvidos para propiciar maior compatibilidade nos compósitos poliméricos [13].

O objetivo do presente trabalho foi avaliar o efeito do tratamento das fibras de bagaço de cana-de-açúcar por explosão a vapor na compatibilização e nas propriedades de compósitos dessas fibras com matriz de poli(tereftalato de etileno) (PET), buscando alternativas o aprimoramento de compósitos poliméricos com fibras vegetais.

A reciclagem de PET aliada a um novo material, sendo este um compósito reforçado com fibra de bagaço de cana-de-açúcar, visa à reciclagem do PET, bem como, aproveitar esse material lignocelulósico, o que ocasiona a substituição de outros materiais plásticos advindos de exploração do petróleo, por materiais sustentáveis e menos agressivos ao meio ambiente.

\section{MATERIAIS E MÉTODOS}

O PET virgem utilizado neste trabalho na forma de grânulos foi doado pela empresa LORENPET, situada na cidade de Lorena/SP, enquanto que o PET reciclado foi obtido a partir de embalagens de bebidas descartadas. As fibras vegetais foram obtidas de bagaço de cana-de-açúcar doadas pela usina Olho D'Água S/A, no Estado de Pernambuco. Também foram utilizados o estabilizante térmico Songnox 21B e detergente de uso doméstico.

Para a preparação dos compósitos de PET com fibra de cana-de-açúcar, as garrafas de bebidas descartadas de PET foram inicialmente lavadas em solução de água e detergente, depois secas em temperatura ambiente e fragmentadas em um moinho de facas da marca MOMESSO instalado no Departamento de Engenharia de Materiais (DEMAR) da EEL/USP.

As fibras de cana-de-açúcar in natura foram obtidas a partir da lavagem com água, secagem a temperatura ambiente e a moagem do bagaço feita no moinho de facas da marca MOMESSO, enquanto as fibras tratadas foram submetidas ao processo de explosão a vapor em um reator com capacidade para $2,5 \mathrm{~m}^{3}$, aquecendo-se a $180^{\circ} \mathrm{C}$ por 15 min. Após o término de reação a válvula é feita a descompressão no reator, provocando 
o processo de expansão brusca das fibras (explosão) e consequente arraste do material para o ciclone acoplado ao reator, no qual as fibras foram lavadas com água para a remoção dos componentes hidrolisados e, na sequência, secas em temperatura ambiente. A preparação inicial das fibras in natura foi realizada no Departamento de Biotecnologia (DEBIQ) da EEL/USP, enquanto o tratamento de explosão à vapor das fibras foi realizado na usina Olho D’Água e no Departamento de Engenharia da Universidade Federal de Pernambuco.

Para a uniformização dos tamanhos e separação de agregados, as fibras foram peneiradas em malha de 20 e 30 mesh. Foram utilizadas as fibras retidas nas peneiras de 30 mesh com comprimento em torno de 2,0 $\mathrm{cm}$ e diâmetro em torno de $0,6 \mathrm{~mm}$.

Antes do processamento termomecânico para a preparação dos compósitos, as fibras e o PET foram secos em estufa de circulação forçada de ar da marca QUIMIS, na temperatura de $120^{\circ} \mathrm{C}$ durante 12 horas. $\mathrm{Na}$ sequência, foi feita uma pré-mistura da fibra com o PET em proporções variadas, conforme apresentado na Tabela 1. Em todas as amostras foi adicionado o estabilizante térmico Songnox 21B na concentração de $0,15 \%$

Tabela 1: Composição e identificação dos materiais.

\begin{tabular}{l|c|l}
\hline AMOSTRA & $\begin{array}{l}\text { TEOR EM MASSA } \\
\text { DE FIBRA NO } \\
\text { COMPÓsITO (\%) }\end{array}$ & IDENTIFICAÇÃO \\
\hline PET virgem & -- & PETv \\
\hline PET virgem após extrusão & -- & PETve \\
\hline PET reciclado & -- & PETr \\
\hline Fibra in natura & -- & Fin \\
\hline Fibra tratada por explosão a vapor & -- & Ftr \\
\hline Compósito com fibra in natura & 5 & F5in \\
\hline Compósito com fibra in natura & 10 & F10in \\
\hline Compósito com fibra tratada & 5 & F5tr \\
\hline Compósito com fibra tratada & 10 & F10tr \\
\hline
\end{tabular}

Após a pré-mistura das fibras com o PET, o material foi submetido ao processamento termomecânico em uma extrusora monorosca da marca IMACOM com rosca que possui elementos de mistura do tipo Maddok e Abacaxi instalada no DEMAR-EEL-USP. O PETv e o PETr foram processados com perfil de temperatura das zonas de alimentação, compressão, mistura e matriz de $200^{\circ} \mathrm{C}, 210^{\circ} \mathrm{C}, 220^{\circ} \mathrm{C}$ e $230^{\circ} \mathrm{C}$, respectivamente, sob rotação da rosca de $30 \mathrm{rpm}$, enquanto para o processamento dos compósitos foi utilizado o perfil de temperatura de $200^{\circ} \mathrm{C}, 215^{\circ} \mathrm{C}, 230^{\circ} \mathrm{C}$ e $225^{\circ} \mathrm{C}$, com rotação da rosca em 40 rpm.

Uma porção dos materiais obtidos após extrusão foi utilizada para a preparação de corpos de prova para ensaios de tração e impacto por moldagem por injeção em uma injetora de termoplásticos DIPLOMAT, modelo SPAZIO DW-130 PLATINUM PLUS, instalada no DEMAR EEL-USP, empregando o perfil de temperatura de $255,250,245,230^{\circ} \mathrm{C}$ da zona de alimentação para o bico de injeção e pressões entre 100 a 110 ton. Outra porção dos materiais extrudados foi utilizada diretamente para as análises de caracterização.

As análises de microscopia eletrônica de varredura (MEV) foram realizadas no DEMAR EEL-USP, em um microscópio eletrônico de varredura LEO 1450 VP com tensão de 20kV. Para a realização das análises, as amostras foram fraturadas em nitrogênio líquido, metalizadas com ouro e analisadas em alto vácuo.

As análises termogravimétricas (TGA) foram feitas em um equipamento NETZSCH, modelo STA $449 \mathrm{~F} 3$ na faixa de temperatura de 20 a $900^{\circ} \mathrm{C}$ com uma taxa de aquecimento de $10^{\circ} \mathrm{C} / \mathrm{min}$ sob uma atmosfera de $\mathrm{N}_{2}$ com fluxo de $100 \mathrm{~mL} / \mathrm{min}$. As amostras foram cortadas em pedaços pequenos com massa em torno de $14 \mathrm{mg}$.

As análises de infravermelho por transformada de Fourier (FTIR) foram feitas em um equipamento Shimadzu IR-Prestige-21, utilizando pastilhas de $\mathrm{KBr}$ e os espectros obtidos com resolução de $4 \mathrm{~cm}^{-1} \mathrm{em} 64$ varreduras. Os espectros do PET e dos compósitos PET/fibra de cana foram normalizados com base na intensidade da banca em $3960 \mathrm{~cm}^{-1}$, relativa aos estiramento da ligação C-H.

Os ensaios mecânicos de tração foram realizados em um equipamento EMIC modelo DL 3000, conforme a norma ASTM D638-10 com célula de carga de $5 \mathrm{kN}$. Foram submetidos ao ensaio seis corpos de provas a uma velocidade de ensaio foi de $5 \mathrm{~mm} / \mathrm{min}$, enquanto os ensaios de resistência ao impacto Charpy 
foram realizados em um equipamento WOLFGANG OFIST modelo RATHENON DDR, com pêndulo para escala de testes de $4 \mathrm{~J}$, no Departamento de Materiais e Tecnologia da Universidade Estadual PaulistaUNESP de Guaratinguetá.

\section{RESULTADOS}

\subsection{Microscopia eletrônica de varredura (MEV)}

A Figura 1 apresenta as imagens de MEV das fibras de cana-de-açúcar in natura e após o tratamento por explosão a vapor, respectivamente. Nota-se que as fibras in natura são compostas por microfibras alinhadas no sentido longitudinal de um feixe que também aparenta ter uma estrutura esponjosa. Por outro lado, as fibras tratadas por explosão a vapor mostram regiões em que as microfibras que as constituem estão emaranhadas e outras regiões em que a disposição longitudinal ainda permanece. A estrutura das fibras tratadas reflete justamente o processo em que a fração polissacarídica da biomassa lignocelulósica é expandida, ocorrendo o rompimento da estrutura de dentro para fora. $\mathrm{O}$ desprendimento das microfibras gera um aumento na área superficial das fibras e deve conduzir, a princípio, a um maior ancoramento e interação com a matriz polimérica, produzindo maior integração das propriedades da matriz polimérica e das fibras da cana-de-açúcar nos compósitos.

a)

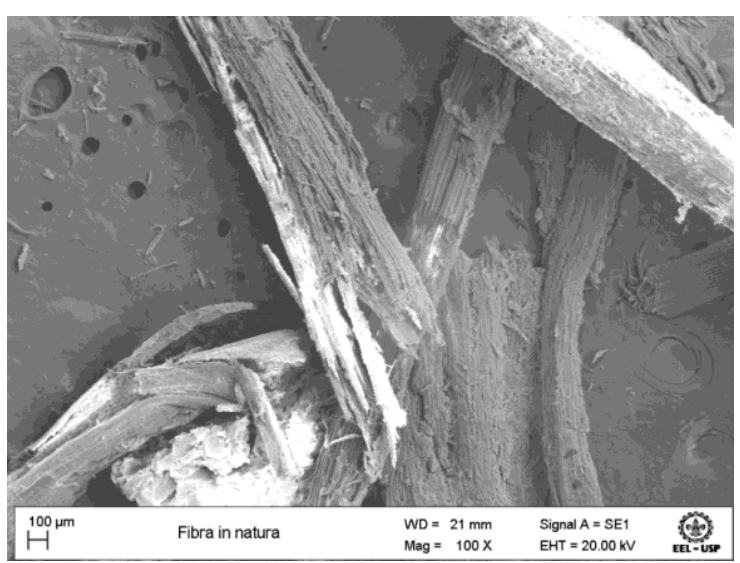

b)

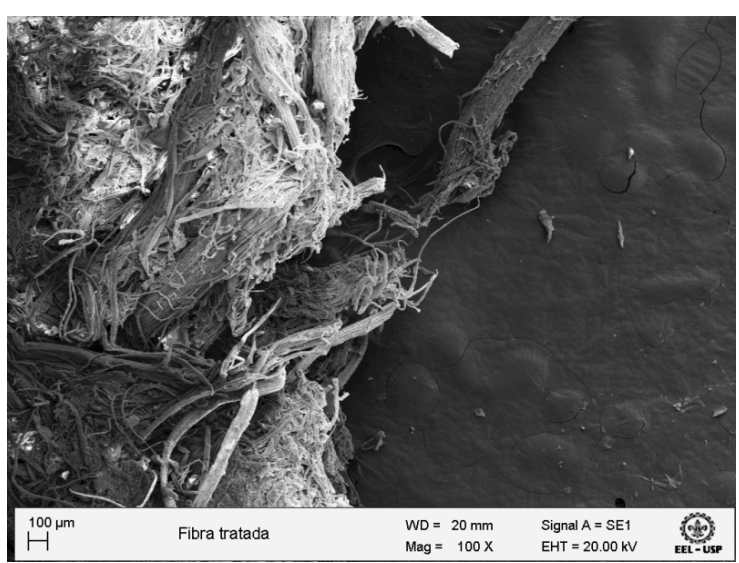

Figura 1: Imagem de MEV de fibra de cana-de-açúcar: a) in natura e b) tratada por explosão à vapor.

Imagens de MEV dos compósitos com 10\% de fibra in natura e tratada por explosão à vapor são apresentadas na Figura 2. Nota-se que as fibras tanto in natura como tratadas apresentam uma boa adesão com a matriz polimérica, não sendo verificados descolamento das fibras em função da fratura criogênica do material. No compósito com fibra tratada (Figura 4) a morfologia permanece similar à do compósito com fibra in natura. Portanto, mesmo com a mudança da estrutura das fibras pelo processo de explosão a vapor não houve penetração da matriz de PET no interior das fibras, o que poderia ocasionar mudanças consideráveis na interação fibra/matriz e nas propriedades do compósito. A alta viscosidade do polímero mesmo no estado fundido ou mesmo as diferenças de polaridade dos materiais podem justificar a dificuldade de penetração do polímero no interior da fibra tratada.

a)

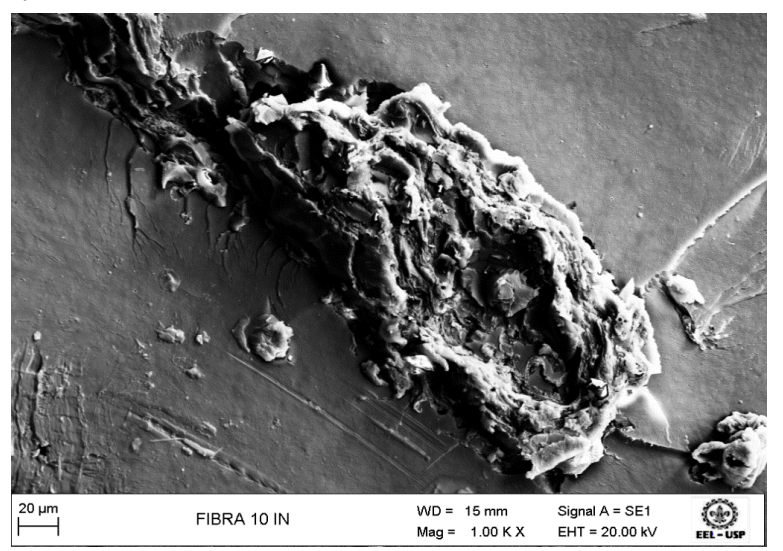

b)

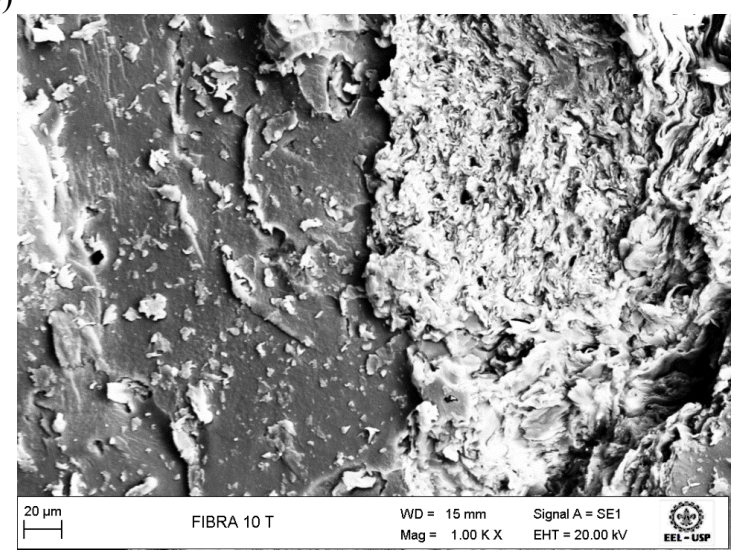


Figura 2: Imagem de MEV de compósito PET/fibra de cana-de-açúcar: a) in natura e b) tratada por explosão à vapor.

\subsection{Análise Termogravimétrica (TGA)}

Os resultados das análises de TGA dos materiais são apresentados na Tabela 2 e na Figura 3. Na Figura 3a é feita uma comparação entre as curvas de TGA das fibras in natura e tratadas, do PET virgem e do PET reciclado, enquanto na Figura $3 b$ encontram-se as curvas de primeira derivada (DTG) dos materiais.

a)

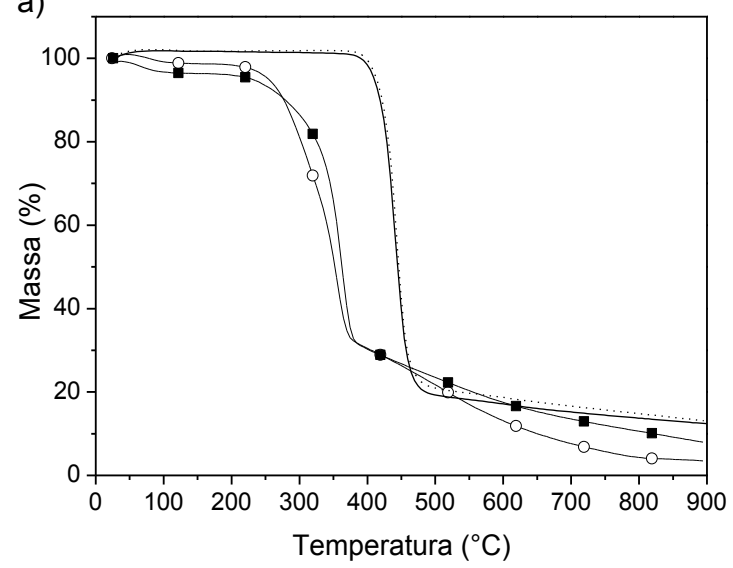

b)

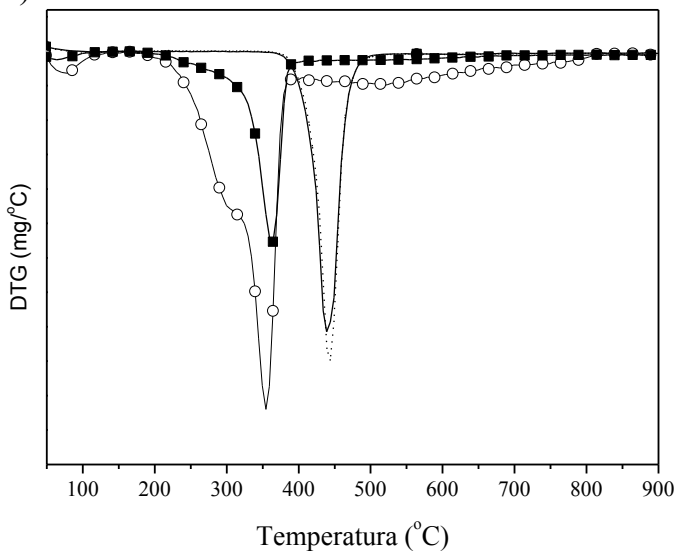

Figura 3: Curvas de TGA do PET e das fibras de cana do PETv (linha sólida), PETr (linha pontilhada), Fin (--) e Ftr (-o-): a) TGA e b) DTG.

Tabela 2: Dados das etapas de decomposição térmica dos materiais.

\begin{tabular}{|c|c|c|c|c|c|c|c|c|c|}
\hline \multirow{2}{*}{ AMOSTRAS } & \multicolumn{2}{|c|}{ ETAPA 1} & \multicolumn{2}{|c|}{ ETAPA 2} & \multicolumn{2}{|c|}{ ETAPA 3} & \multicolumn{2}{|c|}{ ETAPA 4} & \multirow{2}{*}{$\begin{array}{l}\text { MASSA } \\
\text { RESIDUAL } \\
(\%)\end{array}$} \\
\hline & $\mathrm{DT}^{*}\left({ }^{\circ} \mathrm{C}\right)$ & $\mathrm{PM}^{* *}(\%)$ & $\mathrm{DT} *\left({ }^{\circ} \mathrm{C}\right)$ & $\mathrm{PM}^{* *}(\%)$ & $\mathrm{DT} *\left({ }^{\circ} \mathrm{C}\right)$ & $\mathrm{PM}^{* *}(\%)$ & $\mathrm{DT} *\left({ }^{\circ} \mathrm{C}\right)$ & $\mathrm{PM}^{* *}(\%)$ & \\
\hline Fin & $52-106$ & 2 & $217-383$ & 65 & $383-900$ & 29,5 & ----- & ----- & 3,5 \\
\hline Ftr & $42-105$ & 3 & $210-383$ & 65 & $383-900$ & 24 & ----- & ----- & 8 \\
\hline PETV & ----- & ----- & ----- & ----- & $376-490$ & 80 & $490-900$ & 8 & 12 \\
\hline PETr & ----- & ----- & ----- & ----- & $376-490$ & 78,5 & $490-900$ & 8,5 & 13 \\
\hline PETve & ----- & ----- & ----- & ----- & $375-490$ & 80 & $490-900$ & 8.5 & 13 \\
\hline F5in & ----- & ----- & ----- & ----- & $341-484$ & 80 & 484-900 & 19 & 1 \\
\hline F10in & & & $310-367$ & 4 & $367-489$ & 74 & 489-900 & 10 & 12 \\
\hline $\mathrm{F} 5 \mathrm{tr}$ & & & & & $344-488$ & 77 & $488-900$ & 17,5 & 5,5 \\
\hline F10tr & & & & & $310-484$ & 79 & $484-900$ & 20 & 1 \\
\hline
\end{tabular}

* Intervalo de decomposição térmica da etapa; **Perda de massa na etapa

Nos termogramas comparativos entre o PET e as fibras de cana-de-açúcar são identificados diferentes eventos de perda massa. Verifica-se que o PET reciclado apresenta um perfil de decomposição similar ao do PET virgem, mostrando basicamente uma etapa principal de decomposição que tem início em $376{ }^{\circ} \mathrm{C}$ e finaliza em $490{ }^{\circ} \mathrm{C}$ (Tabela 2) e que corresponde à perda de aproximadamente $80 \%$ de massa do material. Após esta etapa o resíduo formado ainda apresenta uma perda menos considerável de $8 \%$, restando ao final da análise 12 e 13\% de resíduo, respectivamente para estas duas amostras. A partir dos termogramas de DTG da Figura 6 verifica-se que a decomposição do PET virgem com temperatura inicial $\mathrm{T}_{\text {onset }}$ em $376^{\circ} \mathrm{C}$ atinge uma taxa máxima de perda de massa em $440{ }^{\circ} \mathrm{C}$, o qual não apresenta diferença significativa com o PET reciclado cuja $\mathrm{T}_{\text {onset }}$ também está em $376{ }^{\circ} \mathrm{C}$ com taxa máxima de perda massa em $444{ }^{\circ} \mathrm{C}$. Desta forma, pode-se afir- 
mar que a decomposição térmica do PET reciclado não difere da decomposição do PET virgem.

No caso das fibras são identificadas três etapas decomposição. Para a fibra in natura a primeira etapa ocorre entre 52 e $106^{\circ} \mathrm{C}$, com perda de $1 \%$ de massa, enquanto na fibra tratada o intervalo de decomposição desta primeira etapa está entre 42 e $105^{\circ} \mathrm{C}$, com perda de $2 \%$ da massa inicial. Esta etapa deve ser resultante da perda de umidade do material [14]. A segunda e principal etapa de decomposição das fibras ocorre entre 217 e $383{ }^{\circ} \mathrm{C}$ para a fibra in natura e 210 e $383{ }^{\circ} \mathrm{C}$ para a fibra tratada, com perdas de massa de 66 e $64 \%$, respectivamente. Esta etapa de decomposição é atribuída à decomposição da hemicelulose, quebra das ligações glicosídicas da celulose e decomposição da $\alpha$-celulose [15]. A última etapa de decomposição no intervalo de temperatura entre 383 e $900{ }^{\circ} \mathrm{C}$ ocorre a uma taxa menor de perda de massa e está relacionada com a decomposição e graftização da lignina. Esse processo tem início mesmo em temperaturas abaixo de $383{ }^{\circ} \mathrm{Ce}$ continua ocorrendo após a decomposição da celulose [15]. O fato da fibra tratada iniciar a decomposição em temperatura inferior à da fibra in natura deve ser reflexo da sua estrutura mais aberta, com maior área superficial e maior capacidade de absorção de calor internamente.

Com relação à taxa máxima de decomposição verificada por DTG, os eventos de perda de massa para a fibra in natura com $\mathrm{T}_{\text {onsets }}$ em 52 e $217^{\circ} \mathrm{C}$ ocorrem nas temperaturas de 74 e $355^{\circ} \mathrm{C}$, respectivamente, enquanto o evento com $\mathrm{T}_{\text {onset }} \mathrm{em} 383^{\circ} \mathrm{C}$ praticamente ocorre em taxa constante de perda de massa. Para a fibra tratada, os eventos com $\mathrm{T}_{\text {onsets }}$ em 42 e $210^{\circ} \mathrm{C}$ ocorrem nas temperaturas de 53 e $364^{\circ} \mathrm{C}$, respectivamente, sendo o último evento com $\mathrm{T}_{\text {onset }} \mathrm{em} 383^{\circ} \mathrm{C}$ também verificado em taxa de perda de massa constante. Estas diferenças também devem ser reflexo da estrutura física assumida pela fibra após o tratamento por explosão à vapor.

Na Figura 4 é feita uma comparação entre as curvas de TGA do PET reciclado após extrusão com os compósitos com teores de 5 e $10 \%$ de fibra

a)

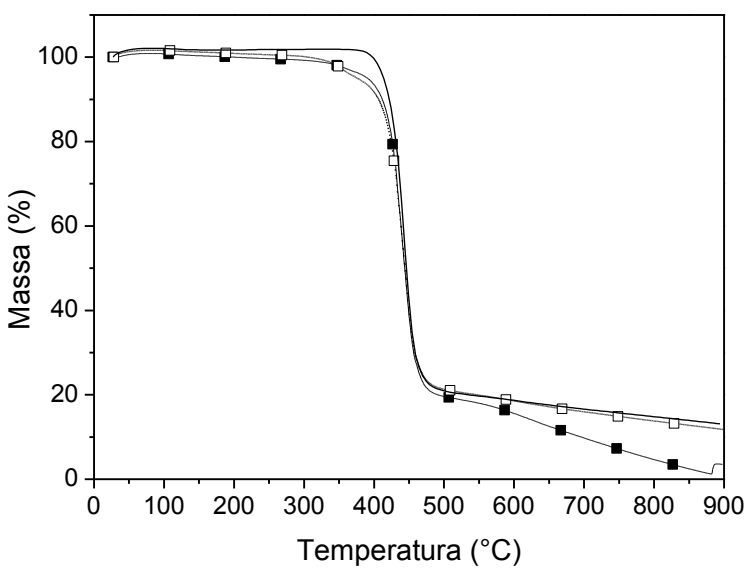

b)

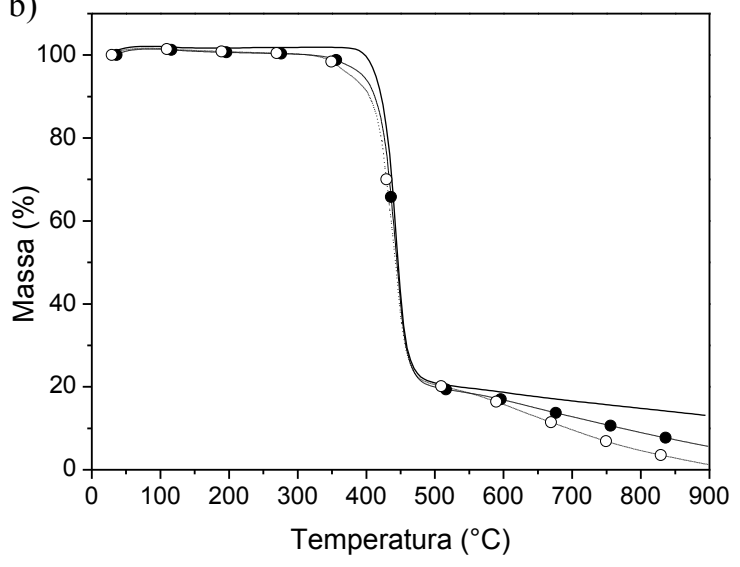

Figura 4: Curvas de TGA do PET reciclado (linha sólida) e dos compósitos F5in (-m-) e F10in (-ロ-): a) com fibras in natura e b) com fibras tratadas.

No compósito com $5 \%$ de fibra in natura (F5IN) o perfil da curva de TGA é similar ao do PETr, apresentado dois estágios de decomposição (Tabela 2). Entretanto, a temperatura em que estes estágios têm início é inferior ao do PETr, principalmente para o primeiro estágio de decomposição. Desta forma, a decomposição inicial do compósito ocorre em uma faixa de temperatura intermediária ao do polímero e da fibra isolados. Outra característica observada é a menor formação de resíduo ao final da análise para esta amostra. Para o compósito com $10 \%$ de fibra in natura (F10IN) podem ser notados três estágios de decomposição térmica. O primeiro no intervalo entre 310 e $367^{\circ} \mathrm{C}$ com $4 \%$ de perda de massa deve ser resultante da decomposição de parte das fibras, embora esteja deslocado para temperaturas maiores quando comparado às fibras in natura isoladas. O segundo estágio também aparece deslocado para temperaturas mais altas quando comparado ao primeiro estágio de decomposição do compósito $\mathrm{F} 5 \mathrm{IN}$, enquanto o último estágio é praticamente igual ao da matriz de PETR isolada. Estes resultados mostram que decomposição térmica dos compósitos de PET com fibra de cana-de-açúcar apresenta um comportamento intermediário ao do PET e das fibras isoladas, sendo que este comportamento é afetado pelo conteúdo de fibra no compósito.

Para o compósito com 5\% de fibra tratada por explosão à vapor (F5T) o comportamento verificado é similar ao compósito correspondente com fibra in natura. Entretanto, não é possível verificar distinção do processo em três estágios como observado no compósito correspondente com fibra in natura. Deste modo, a decomposição que tem início em início em $310{ }^{\circ} \mathrm{C}$ segue até $484{ }^{\circ} \mathrm{C}$, resultando em $79 \%$ de perda de massa. 
Este comportamento de início de decomposição dos compósitos com fibras tratadas em temperaturas mais baixas, tal como nas análises das fibras tratadas isoladamente, deve ser reflexo da própria estrutura gerada nas fibras com o tratamento de explosão à vapor, que proporciona uma maior área superficial nas fibras e consequentemente capacidade de reação com a ação do calor.

\subsection{Espectroscopia no infravermelho por transformada de Fourier (FTIR)}

Na Figura 5 são feitas comparações entre os espectros de FTIR do PET virgem, do PET virgem após o processamento por extrusão, do PET reciclado e das fibras in natura e tratada por explosão a vapor.

Na comparação dos espectros de PET (Figura 5a) são observadas bandas principais que são atribuídas aos grupos químicos presentes na estrutura molecular do polímero. As bandas em torno de $2900 \mathrm{~cm}^{-1}$ são atribuídas ao estiramento da ligação $\mathrm{C}$-H, enquanto a banda centrada em $1700 \mathrm{~cm}^{-1}$ é devida a absorções do grupo carbonila relativas ao movimento de estiramento desta ligação presentes nos grupos ésteres do polímero. Os grupos C-O possuem absorções entre 1000 e $1400 \mathrm{~cm}^{-1}$ e também são observados nos espectros [16]. Nota-se que as bandas acima de $3500 \mathrm{~cm}^{-1}$ nos espectros do PET reciclado (PETr) e do PET virgem após a extrusão (PETve) são ligeiramente mais intensas que no PET virgem antes da extrusão (PETv). Estas bandas estão na região de absorção dos grupos hidroxilas que devem ter sido formadas por processos de degradação durante a sua aplicação, no caso do PET reciclado, e durante o processo por extrusão do PET virgem.

Na comparação feita entre os espectros de FTIR das fibras tratadas e in natura (Figura 5b) não são verificadas mudanças perceptíveis nos espectros, indicando que apesar das mudanças físicas provocadas nas fibras pelo tratamento de explosão a vapor, a estrutura química do material não sofre alterações significativas. As bandas intensas e largas, típicas dos grupos hidroxila (acima de $3000 \mathrm{~cm}^{-1}$ ), e as bandas intensas em torno de $1000 \mathrm{~cm}^{-1}$, que podem ser atribuídas à presença do grupo $\mathrm{C}-\mathrm{O}$, são coerentes com a estrutura química apresentada pelas fibras vegetais.

a)

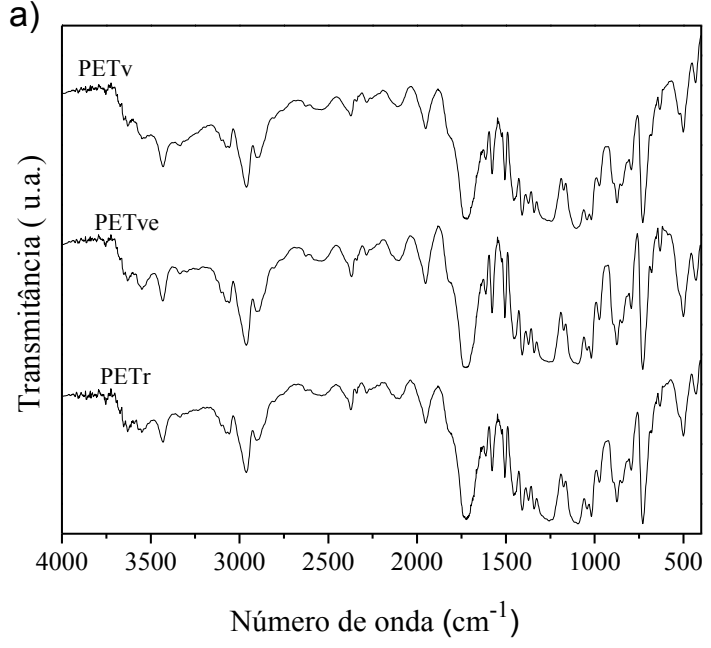

b)

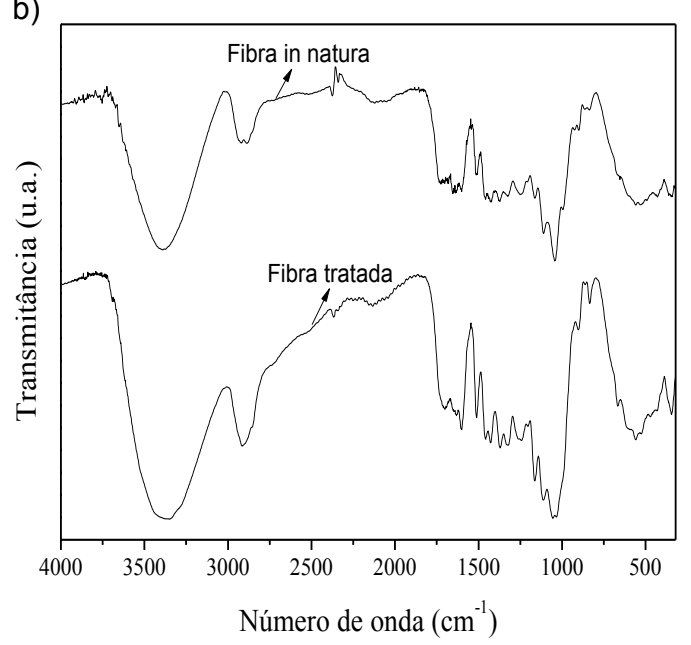

Figura 5: Espectros de FTIR dos materiais: a) PET virgem, reciclado e virgem após extrusão e b) fibras de cana in natu$r a$ e tratada.

Na Figura 6 são feitas comparações entre os espectros de FTIR dos compósitos PET/fibra de cana. Na comparação feita entre os espectros dos compósitos com fibras tratadas e não tratadas com teor de fibra de $5 \%$ pode-se observar diferenças significativas principalmente nas bandas acima de $3000 \mathrm{~cm}^{-1}$ e em torno de $1000 \mathrm{~cm}^{-1}$. Em comparação com os espectros das fibras e do PET isolados (Figura 5), nota-se que espectro do compósito F5in mostra maior semelhança com o espectro da fibra in natura, enquanto o espectro do compósito F5tr apresenta um perfil de bandas mais próximo da matriz de PET. Vale ressaltar que o compósito é um material heterogêneo e durante a realização da análise de FTIR a presença maior de fibras ou da matriz polimérico no trajeto do feixe de infravermelho pela amostra pode resultar a predominância de bandas características de um dos componentes em particular. Já nos espectros dos compósitos com teor de fibra de 10\% (Figura 6b) os espectros apresentam perfis de bandas que são características tanto das fibras como da matriz do PET, sem a predominância evidente de um destes componentes. 
a)

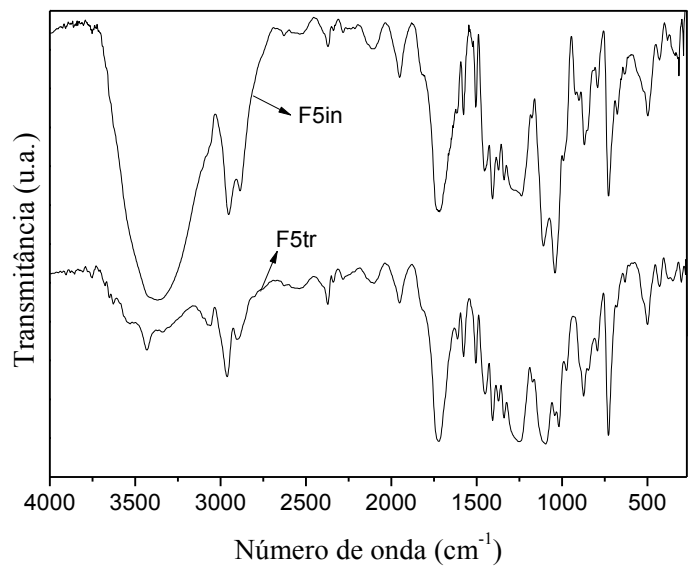

b)

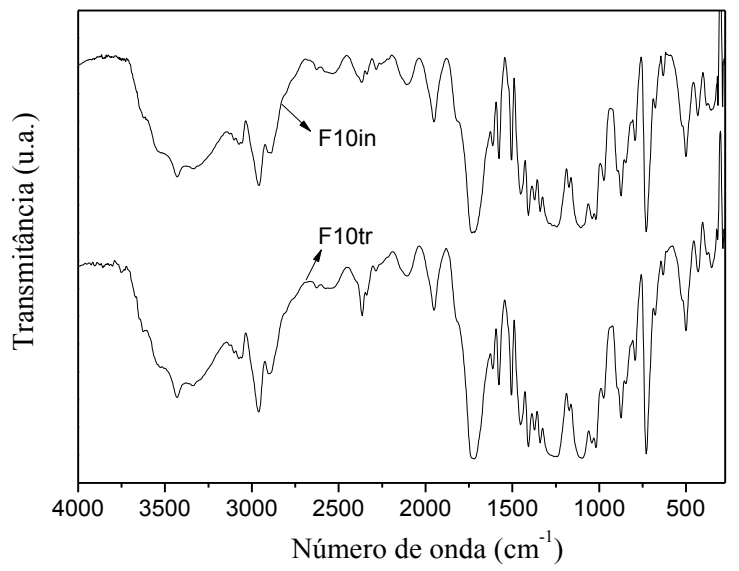

Figura 6: Espectros de FTIR dos compósitos PET/fibra de cana: a) contendo 5\% de fibra e b) contendo $10 \%$ de fibra.

\subsection{Ensaios mecânicos de impacto e tração}

O processamento dos compósitos por meio de moldagem por injeção para a preparação de corpos de prova para ensaios mecânicos apresentou inúmeras dificuldades pelo fato do ajuste das condições necessárias para o processo, como altas temperaturas e pressões elevadas, provocarem a degradação do material, de modo que foi possível obter somente corpos de prova em quantidade e qualidade suficientes para atender às normas do ensaio para o compósito F5tr. As temperaturas utilizadas no processamento termomecânico são determinadas principalmente pela matriz polimérica, que no caso do PET exige temperaturas elevadas para plastificação. Assim, as condições necessárias para a moldagem injeção são incompatíveis com a degradação térmica das fibras de cana-de-açúcar, o que inviabiliza a própria aplicação dos compósitos PET/fibra de cana-de-açúcar em produtos gerados por moldagem por injeção nas respectivas condições. Por outro lado, foi possível obter os compósitos por extrusão, sob uma condição limite para a degradação das fibras, de modo que é possível a aplicação dos compósitos em produtos obtidos por meio de extrusão. Na Figura 7 são comparados os resultados de resistência ao impacto e de módulo de Young do PET virgem, reciclado e após extrusão com o compósito F5tr em que foi possível realizar a moldagem por injeção para a preparação de corpos de prova.
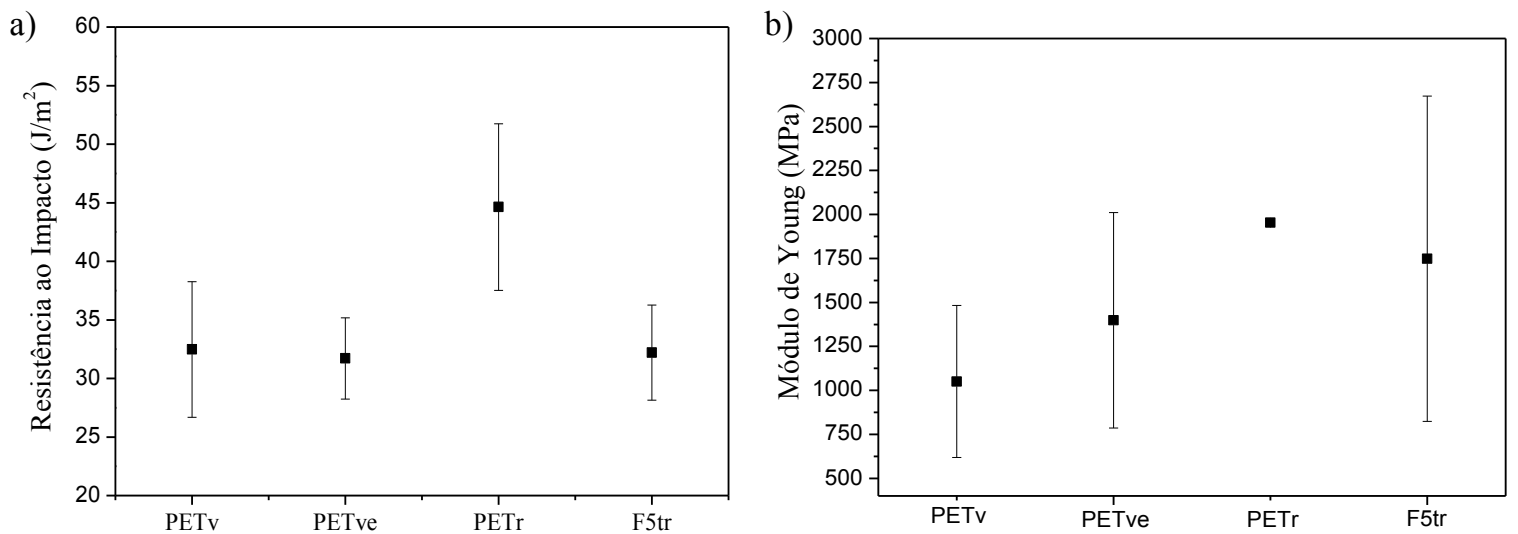

Figura 7: Resultados de propriedades mecânicas do PET e compósito com 5\% de fibra tratada: a) resistência ao impacto e b) módulo de Young.

Observa-se que para a propriedade de resistência ao impacto (Figura 7a), o PET reciclado é o único material que apresenta certa distinção de propriedade com relação aos demais, com valor médio em torno de 45 $\mathrm{J} / \mathrm{m}$, enquanto o PET virgem, o PET virgem após a extrusão e o compósito com $5 \%$ de fibra tratada possuem valores em torno de $32 \mathrm{~J} / \mathrm{m}$. No caso do módulo de Young (Figura 7b), o PET reciclado apresenta uma distinção clara do PET virgem, mostrando o dobro do valor para esta propriedade. Outra característica do PET 
reciclado foi o baixo valor do desvio das medidas, ao contrário do verificado para o PET virgem após extrusão e o compósito com 5\% de fibra tratada, que devido aos valores expressivos de desvio padrão das medidas impedem que seja confirmada alguma tendência nos resultados. Vale ressaltar que a degradação do PET resulta em mudanças progressivas nas propriedades mecânicas do material, tornando-se mais rígido e quebradiço [17], o que está de acordo com os resultados obtidos. Pode-se considerar também que o teor de $5 \%$ de fibra tratada no material não resulta em mudanças expressivas nas propriedades de resistência ao impacto e módulo de Young do PET. Outras propriedades mecânicas obtidas a partir do ensaio de tração como alongamento na ruptura e resistência à tração não apresentaram variações significativas nos valores e não foram consideradas para apresentação.

\section{DISCUSSÃO}

A utilização de fibras vegetais para a preparação de compósitos poliméricos tem sido bastante explorada em pesquisas científicas e também na área industrial, sobretudo automobilística, devido à possibilidade de gerar materiais com custos reduzidos e propriedades suficientes para garantir sua aplicação em diversas situações $[18,19]$. Somando-se a isto, no Brasil há uma grande disponibilidade de fibras vegetais que podem ser utilizadas para a preparação de compósitos poliméricos, como é o caso do bagaço de cana [10]. Um dos pontos fundamentais para a obtenção de bom desempenho das propriedades mecânicas dos compósitos poliméricos com fibras vegetais é conseguir uma interação adequada entre a matriz polimérica e as fibras vegetais [20,21]. $\mathrm{O}$ tratamento prévio das fibras vegetais como de cana-de-açúcar por meio do processo de explosão a vapor provoca um aumento significativo na área superficial das fibras. A utilização deste tratamento, aliado ao emprego de técnicas adequadas de processamento dos compósitos, bem como do uso agentes compatibilizantes, pode resultar na geração de compósitos poliméricos com fibras vegetais que possam atingir o potencial máximo de desempenho de suas propriedades e com custos de produção atraentes para o mercado.

\section{CONCLUSÕES}

O tratamento por explosão a vapor em fibras de cana-de-açúcar promove mudanças significativas na estrutura física do material com o aumento substancial na área superficial das fibras. Entretanto, o processamento termomecânico por meio de extrusão das fibras com o polímero PET para a preparação de compósitos PET/fibra de cana não é capaz de segregar os filamentos gerados com o tratamento por explosão a vapor é inserir a matriz polimérica no interior das fibras, o que poderia resultar em maior compatibilidade no compósito. A utilização de outras técnicas de processamento termomecânico que proporcionam maior cisalhamento do material, como misturadores intensivos, pode ser uma alternativa para alcançar uma morfologia diferenciada no compósito. A geração de produtos por meio da moldagem por injeção dos compósitos PET/fibra de cana-de-açúcar é inviável devido às condições de altas temperaturas e pressões necessárias para o processo que acarretam na degradação das fibras. Entretanto, é possível obter os compósitos e produtos relacionados com o uso do processamento por extrusão sem haver degradação significativas das fibras. A utilização de polímeros termoplásticos reciclados com fibras vegetais de baixo custo para produção de compósitos é uma alternativa que concilia aspectos econômicos, geração de novos materiais e redução de resíduos sólidos.

\section{AGRADECIMENTOS}

Os Autores agradecem à Pró-Reitoria de Graduação da USP pela concessão da bolsa do Programa Ensinar Pesquisa e à FAPESP (proc. 2012/13715-5).

\section{BIBLIOGRAFIA}

[1] Estudo Macroeconômico da Embalagem ABRE/FGV, ASSOCIAÇÃO BRASILEIRA DE

EMBALAGEM, http://www.abre.org.br/setor/dados-de-mercado. Acessado em outubro de 2015.

[2] Indústria Brasileira de Transformação de Material Plástico - Perfil 2014, ASSOCIAÇÃO BRASILERIA DE INDÚSTRIA DO PLÁSTICO - ABIPLAST. http://www.abiplast.org.br.Acessado em outubro de 2015.

[3] JAMBECK, J. R., GEYER, R., WILCOX, C. et al., "Plastic waste inputs from land into the ocean", Science Journals, v. 347, p. 768-771, 2015.

[4] ACHILIAS, D. S., ANTONAKOU, E., ROUPAKIAS, P., et al., "Recycling techniques of polyolefins from plastic wastes", Global NEST J., v. 10, n. 1, pp. 114-122, 2008.

[5] BECKER, D., KLEINSSHIMIDT, A. C., BALZER, P. S. "Compósitos de PVC rígido e fibras de bananeira: Efeito do tratamento da fibra", Matéria, v. 19, n. 3, pp. 257-265, 2014. 
[6] KAYMAKCI, N. A. A., ELMAS, T. A. G. M., "Mechanical performance of composites based on wastes of polyethylenealuminum and lignocellulosics", Composites B, v. 47, pp. 150-154, 2013.

[7] MULINARI, D. R., VOORWALD, H. C., CIOFFI, M. O. H., et al. "Sugarcane bagasse cellulose/HDPE compsites obtained by extrusion”, Comp. Sci. Technol., v. 69, pp. 214-219, 2009.

[8] ROZMAN, H. D., KUMAR, R. N., ADLLI, M. R. M. et al., "The effect of lignin surface activation on the mechanical properties of rubberwood-polypropylene composites", J. Wood Chem. Tecnol., v. 18, n. 4, pp. 471-490, 1998.

[9] BASTTISTELlE, R., VIOLA, N. M., BEZERRA, B. S., et al., "Caracterização física e mecânica compósito de polipropileno reciclado e farinha de madeira sem aditivos”. Matéria, v. 19, n. 1, pp. 7-15 2014.

[10] Cana-de-açúcar, MINISTÉRIO DA AGRICULTURA, http://www.agricultura.gov.br/vegetal/culturas/ cana-de-acucar. Acessado em setembro de 2015.

[11] FARIA, K. C. P., GURGEL, R. F., HOLANDA, J. N. F. "Influência da adição de resíduos de cinzas de cana-de-açúcar nas propriedades tecnológicas de cerâmica vermelha". Matéria, v. 17, n. 3, pp. 1054-1060, 2014.

[12] FENGEL, D., WEGENER, G. Wood: chemistry, ultrastructure, reactions. Berlin, Walter de Gruyter 1989.

[13] GOLDSTEIN, I. S. Wood structure and composition, New York, Marcel Dekker, 1991.

[14] CHETEHOUNA, K., BELAYACHI, N. RENGEL, B. et al., "Investigation on termal degradation and kinetic parameters of innovative insulation materials using TGA-MS”, Appl. Thermal Eng., v. 81, pp. 177-184, 2015.

[15] MANFREDI, L., RODRÍGUEZ, N., WLADYKA-PRZYBYLAK, M., et al., "Thermal degradation and fire resistance of unsaturated polyester, modified acrylic resins and their composites with natural fibres". Polym. Degrad. Stab., v. 91, pp. 255-261, 2006.

[16] SILVERSTEIN, R. M., WEBSTER, F. X. Spectrometric identification of organic compounds. 6 ed. New York, John Wiley \& Sons, 1998.

[17] PUANGSANSUK, K., OPAPRAKASIT, M., UDOMKICHDECHA, W., et al., "Effects of saturated acids on physical properties of UPE resins prepared from recycled PET produts", J. Polym. Environ., v. 17, n. 2, pp.65-70, 2009.

[18] GRISON, K., PISTOR, V., SCIENZA, L.C., et al. "The physical perspective on the solid and molten states associated with the mechanical properties of eco-friendly HDPE/Pinus taeda wood-plastic composites", J. Appl. Polym. Sci. v. 133, n.3, pp.42887-42892, 2016.

[19] IBRAHIN, I.D., JAMIRU, T. SADIKU, E. R., "Mechanical properties of sisal fibre-reinforced polymer composites: a review”. Compos. Interface. v. 23, n.1, pp.15-36, 2016.

[20] ESLAMI-FARSANI, R., "Effect of fiber treatment on the mechanical properties of date palm fiber reinforced PP/EPDM composites", Adv. Comp. Mater. v. 24, n.1, pp. 27-40, 2015.

[21] MAIJA, P., SUVANTO, M., PAKKANEN, T.T., "Cellulose reinforced high density polyethylene composites - Morphology, mechanical and thermal expansion properties”, Comp. Sci. Technol. v. 76, n.1, pp. 21-28, 2013. 